\title{
Neuroscience of apathy and anhedonia: a transdiagnostic approach
}

\author{
Masud Husain ${ }^{1} \&$ Jonathan P. Roiser ${ }^{2}$ \\ ${ }^{1}$ Nuffield Department of Clinical Neurosciences \& Department of Experimental \\ Psychology, University of Oxford. John Radcliffe Hospital, Oxford OX3 9DU, UK. \\ ${ }^{2}$ Institute of Cognitive Neuroscience, University College London, Queen Square, London \\ $W C_{1} N_{3} A R, U K$.
}

Correspondence to M.H. masud.husain@ndcn.ox.ac.uk

\section{Acknowledgements}

M.H. and J.P.R. are supported by fellowships from The Wellcome Trust. M.H. is also supported by the NIHR Oxford Biomedical Research Centre and the Wellcome Trust Centre for Integrative Neuroimaging, Oxford. J.P.R. is also supported by the Leverhulme Trust. 


\begin{abstract}
Apathy and anhedonia are common syndromes of motivation with no established therapies that occur across a wide range of brain disorders. Research using animal models suggests that a useful framework for understanding motivated behaviour lies in effort-based decision making for reward. The neurobiological mechanisms underpinning such decisions have now begun to be implicated in individuals with apathy or anhedonia, providing an important foundation for developing new treatments. The findings suggest that there might be some shared mechanisms between both syndromes. A transdiagnostic approach that cuts across traditional disease boundaries provides a potentially useful means for understanding these conditions.
\end{abstract}

\title{
Introduction
}

Loss of motivation is a common syndrome observed across neurological and psychiatric disorders. In recent years, researchers have identified lack of motivation (also termed amotivation) among substantial proportions of individuals with stroke ${ }^{1}$; traumatic brain injury ${ }^{2,3}$; common neurodegenerative diseases including Alzheimer's disease ${ }^{4,5}$, Parkinson's disease ${ }^{6,7}$ and vascular dementia or small vessel cerebrovascular disease ${ }^{8}$; rarer disorders such as frontotemporal dementia ${ }^{9}$ and Huntington's disease ${ }^{10} ;$ and psychiatric conditions such as major depressive disorder (MDD) $)^{11,12}$ and schizophrenia ${ }^{13,14}$ (Box 1).

Several syndromes associated with diminished motivation have been described. ${ }^{15}$ Historically, these reports arose from a diverse range of medical and psychological experts in the nineteenth century. ${ }^{16}$ Although different terminologies were used for different patient groups - sometimes rather loosely and interchangeably - it is now recognized that these phenomena overlap greatly.

In neurological disorders, amotivation is typically categorised as the syndrome of apathy, defined as diminished motivation for physical, cognitive or emotional activity..$^{17-19}$ In psychiatry, although the term apathy has also been used, amotivation is more often referred to in the context of either anhedonia ${ }^{20}$ or negative symptoms [G] ${ }^{21}$ Classically anhedonia was defined by Ribot as an inability to experience pleasure. This was later broadened in psychiatric diagnostic criteria to include a motivational component - i.e., a loss of interest or pleasure in previously rewarding activities. ${ }^{22}$ Recent research suggests that there might be some common mechanisms underlying both apathy and anhedonia.

Findings from behavioural studies, computational modelling, neuropharmacological or optogenetic manipulations, brain lesions, deep brain stimulation and neuroimaging - in humans and in animal models - have identified the brain systems that are dysfunctional in amotivated states. The results point to disruption of mechanisms underlying the way in which reward is processed to motivate behaviour. ${ }^{22-25}$ In this Review, we explain how, broadly speaking, these mechanisms can be conceptualized within the framework of effortbased decision making for rewards. ${ }^{26,27}$ That is, how the potential benefit or reward for performing an activity is evaluated with respect to the cost in effort required to attain it.

The mechanisms underlying such decision making appear to be affected in people with amotivation across brain disorders regardless of underlying pathology, suggesting that it 
might be timely to develop a transdiagnostic approach which cuts across traditional diagnostic boundaries. Here we examine whether such an approach might indeed be useful by focusing on apathy and anhedonia, and their associated neural and behavioural signatures, across different neurological and psychiatric disorders.

\section{Definitions}

Although there has been debate, most experts now consider apathy to be a syndrome. One set of criteria ${ }^{19}$ builds on previous conceptualizations ${ }^{28,29}$ and has been validated across neurological and psychiatric conditions. It defines apathy as loss of or diminished motivation compared to an individual's previous state; associated with at least two out of three of diminished goal-directed behaviour, cognitive activity or emotion; that causes clinically significant impairment in everyday life; but is not explained by physical or motor disability, reduced conscious level or drugs. ${ }^{19}$ However, it is appreciated that this definition might not capture all aspects of apathy. For example, recent research has identified social apathy - reduced interest in interacting with other people ${ }^{30,31}$ - as another possible component or dimension of the syndrome.

Some clinicians also use the terms avolition ${ }^{21}[G]$ or abulia ${ }^{16}[G]$. People with avolition or abulia encounter difficulty in initiating behaviours, but can perform the same actions when prompted to do so. Avolition can be a prominent negative symptom of schizophrenia. ${ }^{21} \mathrm{An}$ extreme form of avolition is akinetic mutism [G], which is characterized by little or no selfgenerated movement or speech. ${ }^{32}$

Anhedonia is defined as consistently and markedly diminished interest or pleasure in almost all daily activities. Although originally conceptualized as lack of pleasurable experience, for several decades psychiatrists have recognized that this symptom may also reflect a loss of interest to act in order to seek pleasure. ${ }^{22}$ This distinction is important for recent conceptualisations of amotivational syndromes, which consider anhedonia to share some aspects of apathy. It is also important for animal models of anhedonia, in which affective experiences of pleasure are challenging to measure, whereas motivation to obtain rewards is far easier to investigate. Similar to work on apathy, there is evidence that anhedonia might exist for different dimensions, with dissociable axes of loss of interest or pleasure in social activities, sensory experiences, hobbies, or food or drink. 33 Fractionation of apathy and anhedonia into components potentially provides a way to examine similarities between the syndromes at a fine-grain level.

Along with depressed mood, anhedonia is one of the cardinal symptoms of MDD. According to the DSM-5 (Diagnostic and Statistical Manual of Mental Disorders, Fifth Edition) criteria, patients meet criteria for MDD if they have five or more symptoms, one of which must be either depressed mood or anhedonia. However, anhedonia can occur outside of MDD. For example, it has long been recognized as a negative symptom of schizophrenia, and is increasingly appreciated to be an important component of posttraumatic stress disorder, eating disorders and substance use disorder. ${ }^{34}$

Anhedonia and apathy are measured using either questionnaires filled by the patient or caregiver, or by a structured interview performed by the clinician (Box 1). The closeness of 
apathy and anhedonia as syndrome constructs is emphasized by the results of a few studies that have examined both in clinical populations. In Parkinson's disease, for example, some investigators have reported significant positive correlations between scores on apathy and anhedonia scales. ${ }^{35}$ In schizophrenia, apathy and anhedonia usually cluster together as negative symptoms ${ }^{36-38}$, and the severity of apathy correlates with scores of anhedonia, avolition or asociality 39 as well as with negative symptom scores. $4^{\circ} \mathrm{However}$, some reports also show that anhedonia - at least in terms of inability to experience pleasure - can be dissociable from apathy, for example in Parkinson's disease..$^{4}$

These findings demonstrate how important it might be to deconstruct apathy and anhedonia into component processes, rather than to consider them as single, monolithic entities. To the best of our knowledge such an approach, using either behavioural experimental studies or computational modeling, has not hitherto been used to compare mechanisms underlying both apathy and anhedonia within the same individual or patient group (see also Box 1).

Aspects of apathy and anhedonia may also relate to two common symptoms: anergia [G] and fatigue [G]. People with anergia complain of feeling sluggish, being drained or lacking strength even without exerting themselves, whereas fatigue refers to tiredness following activity, either physical or mental. It is not uncommon to find patients who articulate lack of motivation in terms of lacking energy or being fatigued. When apathy, anhedonia and fatigue are evaluated concurrently, there are significant positive correlations between all of these symptoms ${ }^{31}$, for example in Parkinson's disease..$^{42}$ Furthermore, the International Classification of Diseases-10 criteria for MDD include lack of energy or fatigue as a cardinal symptom, in addition to anhedonia and depressed mood.

Anergia and fatigue can also occur in individuals without depression - for example, in systemic illnesses or chronic fatigue syndrome. An important question is whether, in addition to any peripheral muscular factors, there might be central motivational contributions to these symptoms. Similarly, it is now recognized that apathy and anhedonia occur not only in the context of clinical diagnoses but also in the general population, in milder forms, particularly with ageing. 43,44 This realization has led some researchers to investigate whether there might be a neurobiological basis to these symptoms in otherwise healthy individuals ${ }^{45}$, with ageing ${ }^{46-48}$ or in people at high risk of developing depression. 49

\section{Behavioural approaches}

Given the importance and clinical impact of disorders of motivation, several paradigms have been developed to examine such behavioural changes in animal models. ${ }^{25}$ In turn, some of these have led to analogous tests for humans, including various clinical populations. ${ }^{24}$ Broadly speaking, it is possible to consider many of the animal-model tasks as examining one or more of the decision, appetitive [G], consummatory [G] or learning [G] phases of behaviour, although different authors vary in their classification of these phases. ${ }^{20,23,25}$ Despite a lack of complete agreement, one area of consensus is that there might be several possible mechanisms - or combinations of mechanisms - underlying amotivation in people..$^{50}$ This has important implications for treatments. Here we examine 
apathy and anhedonia using the conceptual framework of mechanisms deployed to perform effort-based decision making for rewards (Fig. 1).

Option generation. One important component in effort-based decision making for rewards is the ability first to generate options for behaviour ${ }^{51}$ - whether self-generated or cued by environmental stimuli. Many patients with amotivational syndromes are able to perform behaviours when prompted by others, but experience difficulty in initiating activities themselves. Testing volitional generation of behavioural options is challenging in animals. In humans, it is possible to use tests that require participants to generate as many options as possible for real-life scenarios (for example, "It's a sunny day. What could you do?"). Few studies testing option generation have been performed in clinical populations, but one has shown that the degree of apathy in individuals with schizophrenia correlated inversely with their ability to generate options. $4^{40}$ Another study in Parkinson's disease failed to find a relationship with apathy but did uncover a strong relationship with apathy scores in healthy people. ${ }^{22}$ Option generation tests are very similar to tests of fluency [G], an index of (frontal) executive control, raising the possibility that in some individuals symptoms of amotivation may be driven by executive dysfunction. 53

Decision making leading to option selection. Even if the ability to generate options is intact, individuals might experience difficulty in selecting between possible options especially when a decision made now may affect which decisions are available in the future. Many factors affect such selection, including: subjective valuation of potential outcomes; risk or probability of obtaining the reward (probabilistic discounting); devaluation of rewards due to waiting (temporal discounting); and perception of the effort required to gain the reward (effort discounting). ${ }^{54}$ In Parkinson's disease, people with apathy show reduced sensitivity to rewards 55 , particularly low levels of reward. $5^{6}$ The ability to settle on a choice - rather than vacillate - is also important, especially if options are similar in value, or if there are numerous options. In extreme states of amotivation, any effort might be considered too costly so no action is performed, regardless of the potential reward.

Anticipation. Once an individual has selected an option, they typically experience motivational arousal (evidenced by physiological measures such as heart rate or pupil dilation) in anticipation of action and/or reward. ${ }^{57}$ For example, people normally show anticipatory pupil dilatation that scales with potential reward magnitude in advance of making speeded movements to obtain reward. This pupil response is blunted in some patients with apathy (Fig. 2A,B)..$^{8}$

Action and effort. The initiation, maintenance and invigoration of action together constitute part of appetitive behaviour - for example, locomotor approach of an animal to a potentially rewarding experience, such as when searching for food. Appetitive behaviour has been referred to as a measure of 'wanting' (distinct from 'liking')23, although some caution against the use of subjective terms in reference to animal studies.

Tasks used to study appetitive components of behaviour often measure how much effort an animal is willing to allocate to obtain a reward. For example, in progressive ratio tasks, the number of lever presses a rat has to make to obtain a set reward progressively increases until the animal reaches its 'breaking point' and is no longer willing to exert further effort. ${ }^{25}$ 
Similarly, in a variation of the T-maze, rodents have to decide between scaling a barrier to obtain highly rewarding food versus opting for the low-effort, low-reward food option 59

(Fig. 2C). In humans, effort allocation can be manipulated using the number of button presses, the speed of response or the amount of force exerted to obtain rewards (Fig. 2D-F) - often such tasks use choice behaviour as an indication of willingness to exert effort. ${ }^{60,61,55,62}$

Fewer studies have examined willingness to allocate cognitive effort. Rodents might, for example, have to choose to opt for a highly demanding attention trial (detect a brief illumination) over a low-demand one (detect a prolonged illumination) to obtain a greater reward. ${ }^{6}$ In humans too, researchers have probed willingness to expend mental effort (in tasks that place high demands on attention or working memory) versus physical effort (squeezing tightly on hand-held dynamometers). ${ }^{64,65}$ One important aspect of both cognitive and physical effort tasks is that the highly effortful option must be achievable otherwise any observed changes in decision making could relate to probability discounting, not effort discounting.

A paradigm commonly used to assess 'wanting', originally developed in rodents, is Pavlovian-instrumental transfer [G] (PIT). This involves three stages: Pavlovian (passive) conditioning between an initially neutral stimulus (e.g., a tone or light) and a rewarding outcome (food); instrumental (active, choice-based) association between an action (pressing a lever) and a rewarding outcome; and finally, the PIT phase itself - the presentation of the Pavlovian conditioned stimulus (CS) during instrumental performance (usually during extinction - that is, without delivery of rewarding outcomes). Presentation of the (unrelated) CS causes an invigoration of instrumental responding (PIT effect), and is interpreted as reflecting incentive salience, or 'wanting'. ${ }^{66}$ Human analogues of the PIT task have been developed ${ }^{54}$, with some evidence that the PIT effect is attenuated in depression. ${ }^{67}$

Hedonic impact. Consummatory behaviour refers to the achievement of a goal - for example, eating food. Some refer to this as the 'liking' phase of motivated behaviour. ${ }^{13}$ One probe used to index consummatory behaviour in animal models is the sucrose preference test: rodents given the choice between water and dilute sucrose solution develop a preference for the latter. However, animals exposed to chronic mild stress (a rodent model of depression) show decreased preference for sucrose ${ }^{68}$, taken to indicate reduced hedonic capacity. Direct pleasure from the consummatory phase of behaviour has also been indexed by facial expressions of rodents and primates in response to sweet versus bitter substances. ${ }^{23}$ However, several studies in humans suggest that hedonic aspects of the consummatory phase (measured by self-reported pleasure to sweet tastes) may be intact in people with schizophrenia with pronounced negative symptoms ${ }^{24}$ or $\mathrm{MDD}^{69}{ }^{6}$ raising the question of how appropriate decreased 'liking' (assessed through sucrose preference or facial expression) in rodents is as a model of anhedonia in humans.

Learning. Finally, an important aspect of effort-based decision making for reward is how individuals learn from the outcomes of their actions to guide future selections. This is reinforcement learning - how rewards or losses associated with a stimulus or an action alter subsequent behavious through updates to stimulus value. Learning can be assessed by 
examining how choices change over time in response to feedback. In early work, this was often achieved for example, by analysing performance on early versus late trials, e.g., in the lowa Gambling task [G] ${ }^{70}$, revealing differences between patient groups and healthy volunteers. ${ }^{71}$ However, interpretation of such differences is challenging, as several processes could potentially contribute (e.g., option selection, hedonic impact and learning). A fruitful alternative approach that has gained popularity is to use computational modelling. ${ }^{72}$

\section{Computational modeling}

Computational models (Boxes 2 \& 3) leverage the richness of observed data (e.g., patterns of behaviour which evolve on a trial-by-trial basis) to provide important insight into which processes are important in driving individual differences. $5^{\circ}$ For example, a difficult, partially rewarded perceptual task was developed to examine anhedonia. The authors found reliable, replicable differences in reward responsiveness [G] (indexed by the bias towards selecting the stimulus more frequently associated with reward) between controls and individuals with depression. ${ }^{73}$ However, alterations in several processes - including learning, reward valuation or simply perceptual discrimination - could potentially contribute to such a difference.

Application of a computational model (similar to that in Box 3 ) to a large number of data sets produced a clearer interpretation: symptoms of anhedonia were associated not with differences in incremental learning [G] or perceptual discrimination, but instead with blunted reward valuation (Fig 1). ${ }^{74}$ In a different context, computational modeling of data from a reinforcement learning task in individuals with schizophrenia again revealed an apparent sparing of incremental learning, with poor performance predominantly accounted for by working memory impairment (which can be important in learning tasks due to the time delay between stimuli and outcomes). 75 Thus, the use of computational models in these studies helped to dissect the different cognitive processes involved in reward tasks, showing that learning per se is spared, and that instead differences in behaviour on tests of reward processing are accounted for by different processes.

In Parkinson's disease, computational modeling has demonstrated that improvement in apathy scores when patients were on dopaminergic medication was associated with greater reward sensitivity but not changes in effort sensitivity. 55 Conversely, healthy individuals on a serotonin reuptake inhibitor produced more effort, which modeling revealed to be due to reduced effort costs, not changes in reward sensitivity. ${ }^{76}$ These examples show how using a computational approach can illuminate how specific cognitive processes map on to different symptom profiles and treatments, although to date there has been little systematic use of modeling in this field.

\section{Brain regions}

Reports of previously well people suddenly developing profound amotivation following strokes or other focal lesions have implicated a set of brain regions that appear to be crucial for motivated behaviour in humans. ${ }^{77}$ These include the basal ganglia (particularly ventral aspects including globus pallidus and ventral striatum (VS), parts of the anterior cingulate 
cortex (ACC) and ventromedial prefrontal cortex (VmPFC), which is often referred to as medial orbitofrontal cortex (OFC). . $^{32,78-82}$

Early experimental deep brain stimulation (DBS) studies in people institutionalized for psychiatric disorders reported that they might gain pleasure from self-stimulation of electrodes implanted in the septal region, which included the nucleus accumbens (NAC) part of the VS - and ventral pallidum. ${ }^{83}$ However, closer scrutiny suggests that stimulation might have in fact led to patients merely wanting to engage in more pleasurable activities. ${ }^{84}$ More recent human DBS studies show that ACC stimulation can induce the expectation of an imminent challenge that the patient feels determined to overcome. ${ }^{85}$ Furthermore, DBS of the subgenual ACC can ameliorate symptoms in some people with treatment-resistant depression ${ }^{86}$, although results vary. ${ }^{87}$

Findings from many investigations in animals also converge on a pivotal role in motivation for a network of brain regions that includes ventral basal ganglia structures (such as NAC and ventral pallidum), ACC, ventral tegmental area (VTA) and basolateral amygdala (BLA) (Fig. 3A). ${ }^{26,84}$ The VTA is the source of widespread dopamine (DA) projections to the VS (which represent the mesolimbic DA system); to ACC and prefrontal cortex (mesocortical DA system); and amygdala. In rodents, lesions of NAc or ACC, disconnection of NAc from ACC as well as inactivation of BLA or BLA-ACC connections, can all lead to profound alterations in motivation, with reduced willingness to allocate effort for rewards. ${ }^{88-91}$ In monkeys too, reduced frequency of self-initiated actions to collect reward is observed when bicuculline (a GABA antagonist) is injected into VS and putamen (Fig. ${ }_{3} \mathrm{C}$ ). ${ }^{92}$

By contrast, classical intracranial self-stimulation studies - and more recently optogenetic methods - have revealed that rodents will choose to work in order to receive stimulation of VTA, the medial forebrain bundle (which includes the mesolimbic DA pathway) and parts of the lateral and posterior hypothalamus (Fig 4). 93,94 One pioneering study combined optogenetic stimulation with functional MRI and electrophysiological recordings in rats. Stimulation of MPFC reduced reward-seeking behaviour, assessed for example by sucrose preference. It also blunted striatal activation and reward-seeking elicited by optogenetic stimulation of VTA DA neurons, indexed by the fact that animals that preferred places where they received VTA stimulation no longer did so under simultaneous MPFC stimulation. ${ }^{94}$ The authors consider this to be a potential model for anhedonic behaviour.

Reward and effort valuation signals in humans. A recent meta-analysis has suggested that while vmPFC, VS and midbrain regions invoving VTA might preferentially signal reward; ACC and the anterior insula are key nodes that signal effort. $5^{\circ}$ Activation of ACC and/or the supplementary motor area (SMA) occurs when people perform effort-based decision making tasks. ${ }^{45,95-97}$ One investigation also reported that dorsomedial frontal activation was positively correlated with behavioural apathy scores in healthy people. ${ }^{45}$ This might indicate that people who are more apathetic are confronted by a greater energetic brain cost when making cost-benefit decisions about whether the physical effort required is worth the reward on offer, i.e., when they perform a subjective valuation of a potential reward. Intriguingly, disruption of SMA activity - but not primary motor cortex using transcranial magnetic stimulation also leads to a decrease in perceived effort level. ${ }^{98}$ 
Integration of reward and effort signals. Several neuroimaging studies in healthy humans have attempted to examine the basis of cost-benefit decision making when effort is required to obtain rewards $5^{\circ}$ (Fig 1). Activation of the VS or pallidum varies with reward magnitude and, in some reports, this activity is also modulated by effort, revealing an interaction between reward and effort signals. ${ }^{64,95,99}$ ACC activity is positively correlated with physical effort but negatively related to reward $45,96,100$. Thus some authors argue that the ACC plays a key role in integrating costs (effort) and benefits (reward) to compute the net value of performing an action ${ }^{50}$.

Investigations of cognitive versus physical effort have revealed both common and unique brain activation patterns. ${ }^{64,65}$ One study found that reward devaluation by both types of effort was represented within a common network including ACC and other dorsomedial frontal regions, anterior insula and dorsolateral prefrontal cortex. ${ }^{65}$ Activity within the amygdala appeared to reflect domain-specific processing of the value of rewards associated with cognitive effort. Importantly, activation in the 'domain-general' regions also covaried positively with effort and negatively with reward, suggesting these parameters might be integrated within these areas.

Option selection and option generation. Intriguingly, when people have to select from reward-effort combinations, activation of ACC or SMA corresponds to the difference in reward or effort levels of the chosen and unchosen options. 97 This suggests that these dorsomedial frontal regions might be important for option selection after valuation of potential behavioural options presented to an individual (Fig 1). Which brain regions are involved in self-generated options for behaviour remains to be established but some data point to a role of the pre-SMA and possibly dorsolateral prefrontal cortex. ${ }^{101,102}$

Brain regions in apathy and anhedonia. Neuroimaging studies across a range of human neurodegenerative conditions have revealed that apathy is strongly associated with atrophy of or functional disruption of $\mathrm{dACC}, \mathrm{vmPFC}$ or OFC, VS, VTA and brain regions connected to these areas. ${ }^{27}$ Intriguingly, work examining neural correlates of anhedonia in patients with psychiatric diagnoses has implicated a largely convergent network of brain regions that show blunted activation, relative to controls, when they perform tasks incorporating appetitive, cost-benefit decision and consumatory or learning phases of reward processing. In depression, reduced activation occurs in regions including VS, caudate, vmPFC or OFC and dACC (though contradictory results were reported in this region in different reports). ${ }^{103-105}$

Although the precise pattern of regions observed to showed blunted responses in depression is not identical across studies, some of this inconsistency is attributable to clinical heterogeneity (with frequent reports that the degree of blunting correlates with the severity of anhedonic symptoms ${ }^{103}$ ) and different paradigms. For example, recent investigations reported no differences between depressed and non-depressed groups in reward prediction-error signals in the VS ${ }^{106}$, part of learning from outcomes (Fig 1), in contrast to earlier findings.

The results from investigations in adult depression discussed above are consistent with a large study of motivational processing in adolescents, which identified robust relationships 
between blunted VS activation during the anticipatory phase of reward processing (Fig 1) and symptoms, especially anhedonia (Fig 3B), as well as future risk of depression. ${ }^{107} \mathrm{~A}$ similar picture is evident during the anticipatory phase in studies of anhedonic individuals with schizophrenia ${ }^{108}$, although the ubiquitous use of anti-psychotic medications, which block DA transmission, complicates interpretation. A meta-analysis including 33 studies in depression and 24 studies in schizophrenia (with paradigms tapping a variety of the processes depicted in Fig 1) concluded that reward-related responses were reliably blunted in the caudate, putamen and ACC in both diagnoses. ${ }^{109}$

\section{Neuromodulators}

A large literature exists on the impact of pharmacological manipulations of neurotransmitters on motivational processing in animals. Other investigations have correlated motivation with monoamine neurotransmitter release measured using microdialysis or with fast-scan cyclic voltammetry (FSCV) obtained concurrently with behaviour. ${ }^{110}$ Some studies have manipulated midbrain monoaminergic neurons directly using optogenetic methods. ${ }^{94}$

Dopamine in animal studies. The first evidence for a role of DA in motivation came from findings that depletions of DA - by the neurotoxin 6-OHDA - led to robust reductions in conditioned responses (an aspect of sustaining effort, Fig 1), reversed by amphetamine (which enhances DA signalling). ${ }^{111,112}$ Later, direct microinjections of DA agonists and antagonists into the NAc of rats revealed that DA affects conditioned reinforcement. ${ }^{113}$ Similar manipulations also affect performance on PIT53 and effort expenditure tasks ${ }^{26,114}$ : e.g., DA antagonists reduce vigour (sustaining effort) and preference (cost-benefit decision making) for high effort-high reward options.

Importantly, hedonic responses during the receipt of primary reward [G] (i.e., the consummatory phase of reward processing, sometimes assessed by facial expression, Fig 1) are not affected by DA in rats ${ }^{66}$ but are altered by opiate manipulation. ${ }^{23}$ This suggests that DA profoundly influences the decision and action phases of reward processing, but not the consummatory phase. More recent work shows that adenosine $A_{2 A}$ receptor antagonists can reverse deficits in effort allocation produced by several interventions, potentially by acting on adenosine $A_{2 A}$ receptors that co-localize with $D A D 2$ receptors in striatum and NAc. ${ }^{26}$

There is also good evidence that DA is involved in learning (Fig 1), with an influential study showing that midbrain phasic DA neuron firing corresponds, in timing, to the evolution of reward prediction errors ${ }^{115}$ (a computational quantity indicating the difference between expected and actual outcomes; Box 2). This finding has since been confirmed using optogenetic methods. ${ }^{116,117}$ It led to a proposed distinction between tonic DA, proposed to play a primary role in encoding action and effort, and phasic DA, hypothesised to influence reward learning. ${ }^{114,118}$

However, the dichotomy between tonic and phasic DA has been challenged by FSCV findings. When rats navigate mazes to retrieve rewards, NAC DA signals ramp up slowly during approach as animals come closer to their goals (action and anticipation, Fig 1), 
scaling with reward magnitude. ${ }^{110}$ These DA ramping signals might represent the estimated expected value of reward, which in turn might be used in cost-benefit decisions to evaluate whether it is worth engaging in effortful activity. ${ }^{119}$ Intriguingly, DA release seems to be contingent on action (and therefore effort), not just valuation, because when rats perform a rewarded go/no-go task, DA signals are attenuated when animals must inhibit movement to obtain a reward. ${ }^{120}$

Together, these findings suggest that the tonic-phasic DA hypothesis might require revision, and that instead a unitary account, in which phasic signals simultaneously influence both valuation and learning, might provide a better explanation for the role of DA in reward processing ${ }^{121}$ - with phasic DA signals before choice affecting valuation and propensity to deploy effort, and those during consummation affecting learning. ${ }^{119}$

Dopamine in human studies. Building on evidence linking DA to effort-based decision making for reward in animals, human psychopharmacology has provided a largely consistent set of results. ${ }^{122}$ Dietary depletion of DA precursors, which reduces DA synthesis ${ }^{123}$, attenuates participants' sensitivity to rewards during decision-making ${ }^{124}$ and also increases punishment learning relative to reward learning ${ }^{125}$, with some reporting corresponding reductions in reward-elicited BOLD responses in the striatum. ${ }^{126}$ Research using DA receptor antagonists to block transmission has yielded less consistent results, possibly owing to doses used and the actions of these compounds on other monoamine systems as well as inhibitory autoreceptors. ${ }^{122}$ L-DOPA (which increases DA synthesis), amphetamine and methylphenidate (which block DA reuptake) and D2/3 receptor agonists generally increase a variety of the processes depicted in Fig 1, including: speed ${ }^{127}$ and vigour ${ }^{128}$ of responses (action); effortful ${ }^{61}$ and risky ${ }^{129}$ choices (cost-benefit decision making); reward learning $i^{130}$ and associated reward-related striatal BOLD responses (reviewed in ref 109). ${ }^{122}$

Despite the clear evidence that DA transmission, especially in the striatum, plays a central role in controlling motivated behaviour, standard pharmacological treatments for depression do not target the DA system. ${ }^{131}$ To our knowledge, besides a few pilot studies of agomelatine ${ }^{132}$ (an atypical antidepressant that disinhibits dopamine release through its action on on $5-\mathrm{HT}_{2} \mathrm{c}$ receptors), there has never been a trial specifically focused on treating anhedonia, although a D 2/3 receptor agonist, piribedil, has been reported to be successful in treating of apathy in DBS-treated individuals with Parkinson's disease who subsequently have their DA drug dose reduced. ${ }^{133}$ Dopaminergic drugs also modulate effort-based decision making in Parkinson's by boosting reward sensitivity (cost-benefit decision making, Fig 1). ${ }^{55,56} \mathrm{~A}$ cholinesterase inhibitor has also been reported to confer improvement in apathy in Parkinson's disease. ${ }^{134}$

Interestingly ketamine which antagonises NMDA receptors and is effective in treatmentresistant depression (in which anhedonia is common) (135 $^{13}$, has profound facilitatory downstream effects on DA transmission. ${ }^{136}$ Ketamine has also been reported to be effective in ameliorating anhedonia over-and-above general depressive symptoms, with reduced anhedonia correlating with altered resting-state metabolism in striatum and ACC. ${ }^{137}$ Further studies are needed to investigate whether ketamine can improve symptoms amotivation in disorders other than treatment-resistant depression. 
A few studies have linked DA to motivational symptoms in individuals with neurological and psychiatric disorders using positron emission tomography (PET). In depression, striatal $D_{2} / 3$ receptor binding is reported to be negatively associated with anhedonia. ${ }^{138} \mathrm{In}$ Parkinson's disease ${ }^{139}$ and anhedonic depression ${ }^{140}$ there is a negative relationship between striatal DA transporter binding and amotivation, and in cannabis users apathy has been associated with low DA synthesis capacity. ${ }^{141}$ These attenuations of DA function may in part be caused by chronic inflammation ${ }^{142}$, which is common in disorders in which fatigue, anhedonia and apathy are prominent. ${ }^{143}$

Serotonin in animal studies. The other major neurotransmitter linked to motivation is serotonin ( $5-\mathrm{HT})$. A common finding from neurophysiological studies is there are opponent interactions between $5-\mathrm{HT}$ and DA. ${ }^{144}$ For example, drugs acting at the $5 \mathrm{HT}_{2} \mathrm{C}$ receptor can modulate release from DA neurons of the mesolimbic and mesocortical pathways. The 5$\mathrm{HT}_{2} \mathrm{C}$ receptor antagonist SB-242084 increases the number and duration of effortful responses that rats will make for food rewards (which could be due to changes in either the decision-making or action phases of reward processing, Fig 1). ${ }^{145}$

One influential computational account - based in part on evidence that 5 - $\mathrm{HT}$ plays a role in inhibition of action in the face of aversive stimuli ${ }^{146}$ and in learned helplessness ${ }^{147}$ (which would have the effect of reducing motivational responding) - has hypothesized that DA signals prediction errors for reward, whereas 5-HT signals those punishment (i.e., part of learning, Fig 1). ${ }^{148}$ More recent theoretical formulations propose a more nuanced picture, suggesting that $5-\mathrm{HT}$ may promote Pavlovian (reflexive) inhibitory control (which could be related to either decision making or action). ${ }^{149}$

Empirical research has suggested that a simple relationship between $5-\mathrm{HT}$ and aversive processing is an over-simplification. ${ }^{150}$ Recordings made directly from neurons in the dorsal raphe nucleus (DRN), a major source of $5-\mathrm{HT}$ neurons, show that firing is modulated by the size of upcoming reward (related to anticipation, Fig 1), similar to the pattern observed in DA neurons ${ }^{151}$, though with substantial heterogeneity between DRN neurons, not all of which are serotonergic. Optogenetic stimulation of identified $5-\mathrm{HT}$ neurons results in increased patience for rewards delivered after a delay (part of decision making, Fig 1). ${ }^{152}$ Other optogenetic studies stimulating DRN neurons report that stimulation is reinforcing and elicits a greater propensity to exert effort (part of decision making, Fig 1). However, this effect is not solely related to $5-\mathrm{HT}$ release as some of the stimulated neurons are glutamatergic. ${ }^{153}$

Serotonin in human studies. Pharmacological manipulations of 5-HT in humans have provided some consistent effects. Dietary 5-HT-precursor depletion reliably results in reduced behavioural inhibition in the face of potential punishment (i.e., disinhibition, which would have the effect of increasing vigor, Fig 1) ${ }^{154}$, a conclusion supported by neuroimaging. ${ }^{155}$ Other reports show that precursor depletion reduced representation of expected reward value (assessed using computational modelling of choices during a learning task, part of decision making, Fig 1) ${ }^{156}$ and speed of responding for reward (part of action, Fig 1). ${ }^{157}$ Another investigation in healthy volunteers demonstrated that selective serotonin reuptake inhibitor administration affected decision making, reducing effort costs 
but not reward valuation (Fig 1)..$^{6}$ Of note, apathy in Parkinson's disease has been associated with low $5-\mathrm{HT}$ transporter binding, which some researchers interpret as a measure of neuronal integrity. ${ }^{158}$ However, in general the role of $5-\mathrm{HT}$ in motivation is far from clear and requires substantial further empirical work.

Summary. Extensive investigations have suggested that DA and $5-\mathrm{HT}$ systems play a modulatory role in several aspects of effort-based decision making for rewards. However, the findings do not suggest that there is a simple mapping of each of these neurotransmitter systems to the components identified from behavioural studies (Fig 1). Rather, the results appear to suggest more complex involvement of these neuromodulators, and very few studies to date have examined their interaction in shaping motivated behaviour. These considerations have implications for development of new therapies for apathy and anhedonia. Given the complexity of each behavioural syndrome with dissociable component processes and potentially different patterns of behavioural deficit across individuals labelled as having the same syndrome - it seems unlikely that a single drug therapy would be appropriate for all patients.

\section{Conclusions}

This Review of diverse sets of data from human and animal model experiments reveals some of the mechanisms that might be involved in the genesis of apathy and anhedonia, and provides a framework for developing potential treatments. To move forward, it seems crucial to establish which underlying brain mechanisms contribute to a syndrome of amotivation, such as apathy or anhedonia, in an individual patient. Using clinical definitions might not be sufficient to capture these (see also ${ }^{24}$ ) because different constellations of disrupted mechanisms might occur in different individuals. Moreover, the pattern of deficits need not be disease specific. Hence, future research will need to focus on elucidating the mechanisms that underpin a behavioural syndrome.

As we have seen, anhedonia and apathy are strongly related across different disorders when both have been measured using clinical scales.35,38,39 However, there is not a perfect overlap, so the question arises as to which mechanisms might be common to both and which might be unique to each of these syndromes. The development of new questionnaires provides one way to address this question. One of them now attempts to distinguish between anticipatory and consummatory aspects of motivation but has not always been useful in making distinctions in clinical groups. ${ }^{159}$ However, it has to be borne in mind that these are subjective assessments which do not necessarily provide the granularity required.

Perhaps a better way to answer establish whether there are any fundamental differences between apathy and anhedonia might be to fractionate behaviour and examine underlying components, such as those delineated in Fig 1. To improve phenotyping in this way would require a battery of behavioural paradigms, ideally combined with computational modelling (Boxes 2 and 3 ). To investigate whether dysfunction of the same brain regions underlies the disruptions of reward-related behaviour observed across brain disorders would require the application of a core battery of functional imaging tasks across clinical diagnoses. 
Although both apathy and anhedonia are clinically debilitating and have a profound impact on quality of life, there have been few attempts to develop pharmacological treatments. ${ }^{131}$ This seems an area ripe for investigation, given the clear clinical need and close correspondence between behavioural tests developed to assess humans and animals. The results of pharmacological modulation and stimulation in rodent models have revealed the complexity of neurotransmitter involvement in motivated behaviour. Nevertheless, they provide hope that it might be possible to develop treatments targeted to components of effort-based decision making for reward. This might be relevant to psychological therapies as well. For example, behavioural activation therapy [G] for depression, which specifically targets goal-directed behaviour ${ }^{148}$, can be as effective as cognitive behavioural therapy [G]. ${ }^{160}$ Additionally, a number of psychosocial interventions, including cognitive behavioural therapy or exercise therapy, seem to be modestly effective at treating negative symptoms in schizophrenia, although better-controlled studies are required. ${ }^{161}$

These considerations suggest that to progress further in this field, it is essential to understand the mechanisms underlying the 'surface manifestations' of apathy and anhedonia - the clinical presentation - and characterize the phenotype more carefully in individual cases. The ultimate aim would be to use this framework to develop personalized treatments - pharmacological or psychological - for patients suffering from debilitating motivational symptoms. 


\section{Glossary definitions}

Avolition: Inability to perform self-directed, purposeful activities.

Negative symptom: Thoughts, feelings, or behaviours normally present that are absent or diminished.

Akinetic mutism: Loss of ability to self-initiate limb movement and speech.

Abulia: Reduced spontaneous verbal, motor, cognitive and emotional behaviours.

Anergia: Loss of energy.

Fatigue: Weariness or diminished ability following mental of physical activity.

Appetitive: Approach or goal-seeking phase of behaviour.

Consummatory: Completion or consummation phase of a behaviour.

DSM-IV field trials: Reports on the first attempts to apply the new diagnostic criteria laid down in the Diagnostic and Statistical Manual of Mental Disorders-IV in real-world settings. Learning: Acquisition of information, in this case to alter future behaviour.

Fluency: The number of examples generated of a verbal (e.g. words beginning with the letter F) or non-verbal category (e.g. different patterns on a dot array using four straight lines).

Inverse temperature parameter: Constant term in the commonly used softmax decision rule, a form of the logistic function (see Box 2). The parameter affects the steepness of the function around the inflection point, resulting in more consistent choices at higher values.

Pavlovian-instrumental transfer: The influence of an irrelevant conditioned stimulus on ongoing instrumental behaviour.

lowa Gambling task: A neuropsychological test of decision-making for reward.

Reward responsiveness: The development of a bias towards a more frequently rewarded stimulus.

Incremental learning: Learning over trials.

Primary reward: Rewarding stimuli that facilitate survival of an organism or its offspring, e.g., food, water and sex.

Behavioural activation therapy: A psychological therapy that focuses on activity scheduling to encourage patients to approach activities that they avoid and on analysing the processes (e.g. rumination) that serve as a form of avoidance.

Cognitive behavioural therapy: A psychological therapy that aims to assist a person to change their thinking and behavior by practicing effective strategies to decrease symptoms and distress

Overfitting: Overfitting a model occurs when it becomes too complex (has too many parameters) and begins to describe random error in the data rather than the relationships between variables. 


\section{Box 1 | Measurement and prevalence of apathy and anhedonia}

Clinically, apathy and anhedonia are measured using either self-report questionnaires or structured interviews. ${ }^{13,17-19,162}$ Popular anhedonia questionnaires ${ }^{13,162}$ include the Scales for Physical and Social Anhedonia (which focus on lifetime hedonic responses to specific environmental stimuli), the Snaith-Hamilton Pleasure Scale (more general, less culturally bound questions, rated over recent days) and the Temporal Experience of Pleasure Scale (which attempts to distinguish hedonic and appetitive components of anhedonia). Several questionnaires for apathy exist, with two commonly used ones being the Apathy Evaluation Scale and the Apathy Scale. ${ }^{17-19}$

In interviews, scores are commonly derived using questions from measures such as the Scale for the Assessment of Negative Symptoms in schizophrenia, though such scales usually feature only a small number of items specifically relating to amotivation. ${ }^{13}$ However, structured interviews specifically focused on apathy (such as the Lille Apathy Rating Scale) and anhedonia (Clinical Assessment Interview for Negative Symptoms; Brief Negative Symptom Scale) have been developed. ${ }^{163}$

Estimates of apathy in neurological disorders vary depending on the scales used and selection criteria, but some reported mean prevalence rates are shown in the table below. Good data regarding anhedonia in some of these groups is sparse, largely because studies in neurological disorders focus on apathy. However, in one investigation conducted in Parkinson's disease, almost all patients who were apathetic were also anhedonic, but almost a third of the sample fulfilled criteria for anhedonia without having apathy. ${ }^{164}$

\begin{tabular}{|l|c|c|c|c|}
\hline Disorder & $\begin{array}{c}\text { Apathy in } \\
\text { population (\%) }\end{array}$ & Ref & $\begin{array}{c}\text { Anhedonia in } \\
\text { population (\%) }\end{array}$ & Ref \\
\hline Alzheimer's disease & 49 & 4 & 61 & 5 \\
\hline Frontotemporal dementia & 72 & 9 & $?$ & \\
\hline Huntington's disease & 47 & 10 & $?$ & \\
\hline Major depressive disorder & 38 & 12 & 37 & 11 \\
\hline Parkinson' disease & 40 & 6 & 46 & 7 \\
\hline Schizophrenia & 47 & 14 & 45 & 11 \\
\hline Stroke & 36 & 1 & $?$ & 3 \\
\hline Traumatic brain injury & 61 & 2 & 22 & \\
\hline \multicolumn{1}{|c|}{ Vascular dementia } & 65 & 8 & $?$ & \\
\hline
\end{tabular}

Anhedonia is extremely common in depression, with both population-based surveys ${ }^{165}$ and DSM-IV field trials [G] ${ }^{37}$ suggesting a prevalence of $85-95 \%$ in MDD, based on responses to a single question. Studies using questionnaires (with cut-offs defined by the authors of the scale) report clinically significant anhedonia in $37 \%$ of MDD patients. ${ }^{11}$ In schizophrenia, anhedonia has been reported to vary in prevalence from $45 \%$ when assessed using questionnaires ${ }^{11}$ to $~ 80 \%$ using clinical interviews. ${ }^{13}$ Anhedonia and apathy can occur within the same individual, as reported in both schizophrenia39 and Parkinson's disease. ${ }^{35}$ 
Apathy has a severe impact on the quality of life of both the patient and the carer, as well as on functional independence and prognosis. ${ }^{18}$ The severity of anhedonia varies highly and correlates with more severe symptoms, poorer treatment response and low self-reported quality of life in depression ${ }^{39}$, and poor functional outcome in schizophrenia. ${ }^{166}$

\section{Box 2 | Utility of computational modelling of behaviour}

A computational approach can provide useful insights into what may drive observed patterns of behaviour. Computational models have increasingly been applied to tasks assessing motivation and decision making. In contrast to standard ("descriptive") data analysis, computational approaches start by creating a generative model which includes the processes thought to be involved in task performance, specified in a mathematically precise form (an example is shown in Box 3). Models usually contain a number of free parameters, each of which governs the influence of a specific process on information processing and behavioural output. Parameters are estimated from the data, and can often capture patterns that would not be immediately evident from a descriptive analysis (for example patterns that evolve over time during learning). The estimated parameters can be used to summarise the data alongside traditional descriptive measures, as they can distil large amounts of information and are hypothesised to relate to specific cognitive processes. ${ }^{167} \mathrm{It}$ is also possible to generate uncertainty estimates for each parameter at the individual subject level.

\section{Model fitting}

Model fitting involves adjusting the parameter values in the model until the pattern of output (e.g., choices) it makes matches, as closely as possible, those made by the real participant. ${ }^{168}$ The estimation process also produces a measure of model fit. Often, several different model architectures will be compared (model selection), with parameters extracted from the winning model. To avoid overfitting [G], the fit of a model is balanced against its complexity (related to the number of free parameters). It is also possible to perform averaging of parameters across several models, which can be useful if data from different individuals are best fit by different models (though this only makes sense if models are structurally similar). A crucial process is model checking - that is, testing whether the model faithfully reproduces the observed data - which may, in turn, lead to the construction of a better model. It is also important to check how accurately parameters can be recovered; this involves generating data from the model using known parameters, which should then be estimable. If parameter recovery fails, the model requires revision.

\section{Limitations of the computational approach}

There are certain limitations of the computational approach to consider. ${ }^{169}$ First, although modern desktop computers implement simple models and estimation methods quickly (e.g., maximum likelihood), more complex procedures (e.g., sampling) can be slow and thus not very practical. Second, parameters can be interpreted in multiple ways. For example, the inverse temperature parameter ( $\beta$ in Box 3 ) of the softmax function can be interpreted in terms of consistency, exploration or valuation, limiting the interpretation of such models. 
Third, even simple models can contain redundant parameters, which can result in poor estimation. Fourth, as with any statistical analysis, larger data sets will tend to favour more complex models. This limits the utility of model comparison, as which model is favoured will depend in part on the amount of data included in the model. Indeed, some investigators eschew model comparison altogether, advocating a model checking approach. ${ }^{170}$

\section{Box 3 | Example of a simple computational model}

The example below assumes that participants perform a task in which two stimuli, A and B, are associated with high and low probabilities of reward, respectively. ${ }^{171}$ Participants must learn the contingencies and select the high-probability reward stimulus to perform well. A common way of modelling this type of task is to specify a learning rule (how stimulus or action values are updated over time) and a decision rule (how decisions are made based on those associations).

\section{Learning rule (value updating)}

Learning rules are often based on the prediction error - the discrepancy between actual and expected outcomes ${ }^{115}$ :

$$
\delta=r(t)-v(t)
$$

$\delta$ is the prediction error, which can be positive or negative (better- or worse-than-expected, respectively). $r(t)$ is the reward outcome (0 or 1 ) received on trial $t . v(t)$ is the value of the choice (which depends on the strength of stimulus-outcome association and thus varies over the experiment), which evolves over time (depicted in part a of the Figure). The model then updates the value:

$$
v(t+1)=v(t)+\alpha^{*} \delta
$$

The stimulus value for a new trial is equal to its value on the previous trial, plus the prediction error $\delta$ weighted by $\alpha$, the learning rate parameter. In other words, $\alpha$ specifies the influence of the prediction error. It can be interpreted as the speed of learning, or the influence of recent outcomes - the higher the $\alpha$ value the more recent outcomes influence the value. Part a of the figure shows the effect of varying the value of $\alpha$ (different coloured lines) on value learning over 50 trials, of which $80 \%$ are rewarded and $20 \%$ not rewarded (at identical points in the game).

\section{Decision rule (choosing options based on value)}

When modelling decision making, one possible assumption would be that the higher value stimulus is always chosen. However, in reality organisms rarely behave in this manner and instead occasionally explore low-value options. One decision rule that incorporates the occasional opting for the lower-value option uses the softmax function ${ }^{172}$ :

$$
p(A)=\exp \left(\beta^{*} v_{A}(t)\right) /\left(\exp \left(\beta^{*} v_{A}(t)\right)+\exp \left(\beta^{*} v_{B}(t)\right)\right)
$$


$p(A)$ is the probability of choosing $A$, proportional to the value difference between option $A$ and option $B$. The inverse temperature parameter [G], $\beta$, governs how consistently the model chooses the higher-value stimulus. The higher the $\beta$ value the more deterministically the algorithm chooses the higher value option (or alternatively, the less exploratory its choices), especially when the values are similar. Part $\mathbf{b}$ of the figure shows the effect of varying the value of $\beta$ (coloured lines) on choices between options that are close (centre of $\mathrm{x}$-axis) or very different (extremes of $\mathrm{x}$-axis) in value.
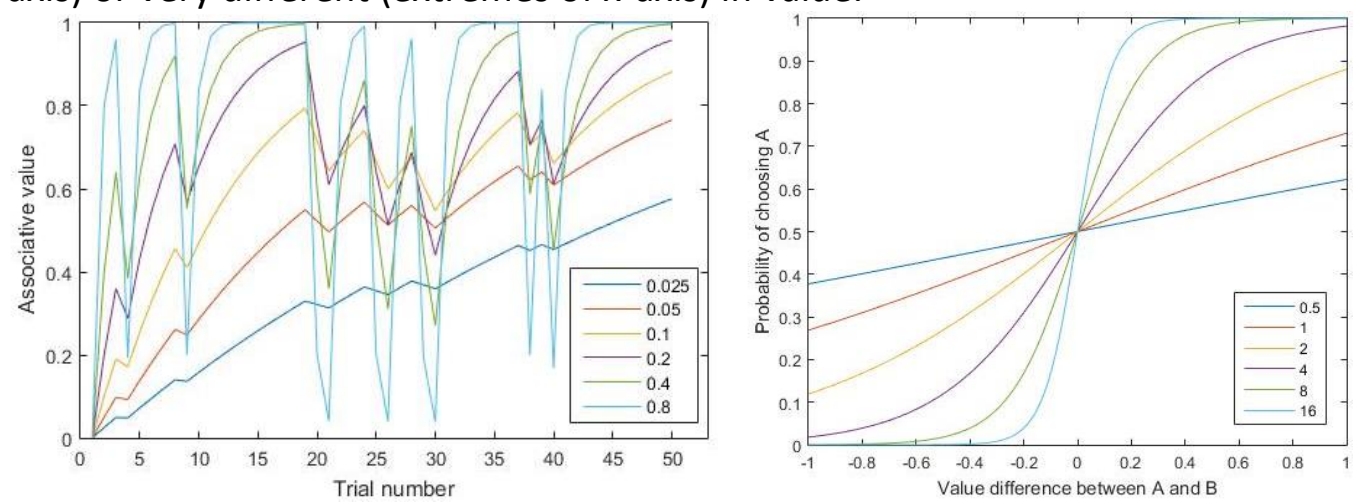


\section{Syndrome}

\section{Apathy Anhedonia}

\section{Behavioral component}

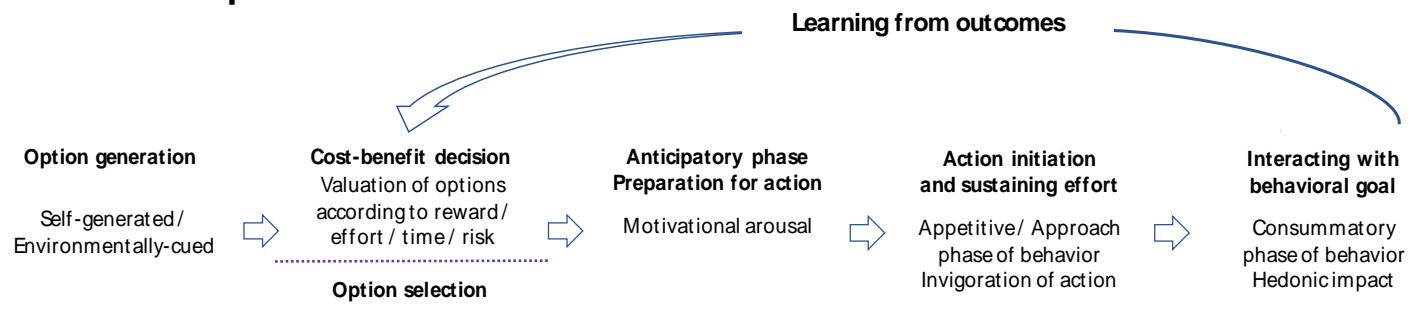

Figure 1 | Mechanisms underlying effort-based decision making to obtain rewards The clinical syndromes of apathy and anhedonia appear to overlap, sharing some possible underlying mechanisms. In the field of effort-based decision making for reward, several possible processes have been identified, some of which are displayed here. 


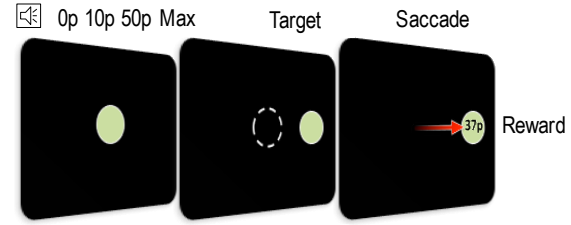

Auditory cue

B

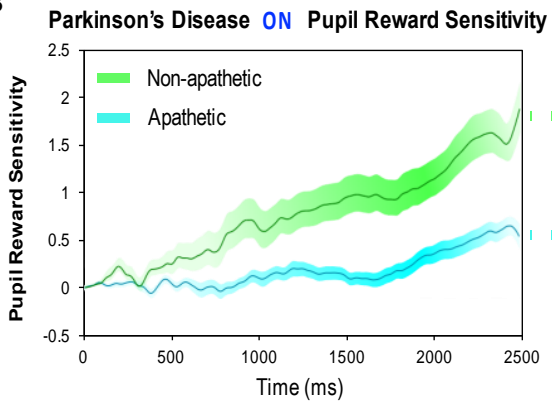

c

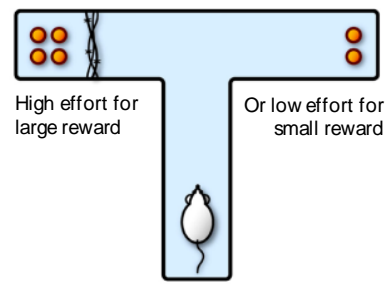

D

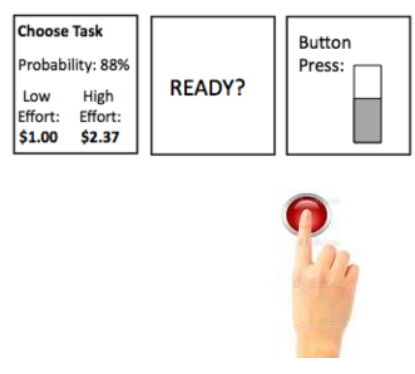

E
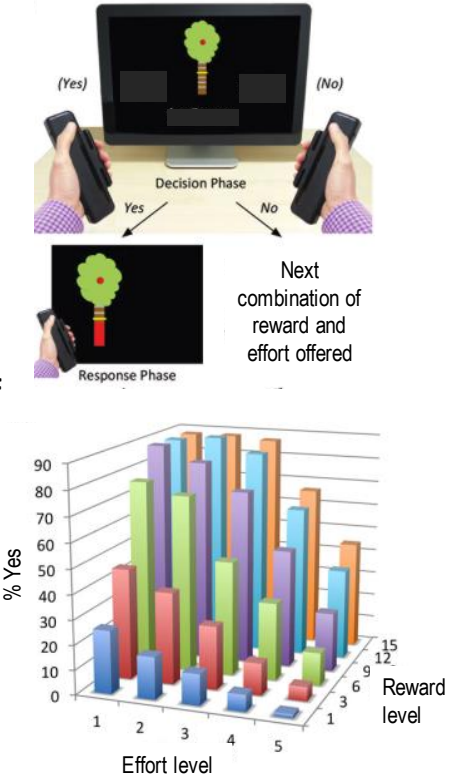

Figure 2 | Behavioural paradigms for assessing amotivation

A Speeded saccade for reward task in which the monetary reward won depends on the speed of response. The reward on offer is announced at the beginning of each trial, before the saccade target is presented. B On this task, participants' pupils normally dilate more with greater anticipated reward after the auditory cue announcing maximum reward on offer. Reward sensitivity of the pupils is blunted in individuals with Parkinson's disease and apathy, compared to those without apathy..$^{58} \mathrm{C}$ Rodent T-maze experiments reveal that depletion of NAC dopamine or lesions of ACC shifts rats from a strategy of working hard for large rewards (scaling an obstacle to obtain the large reward) to opting for a small reward which requires far less effort. 59,89 D Effort task which requires human participants to select between low or high physical effort options (number of button presses) associated with different levels of reward. ${ }^{60} \mathrm{E}$ Effort task where human participants decide to accept or reject offers in which different levels of reward are available for different levels of physical effort (grip force). The image on the screen displays the reward on offer (depicted as the number of apples on a tree) and the force required to obtain that level of reward (indicated by yellow line on tree trunk). F On this task, the likelihood of accepting offers increases with stake (reward on offer) and decreases with increasing effort required. 45 


\section{A}

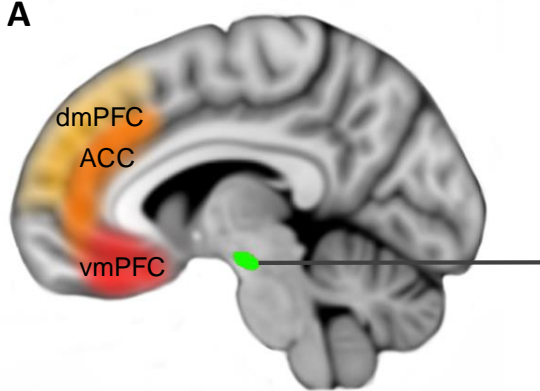

Medial prefrontal regions connected to basal ganglia

Ventral tegmental area (VTA) Substantia nigra complex

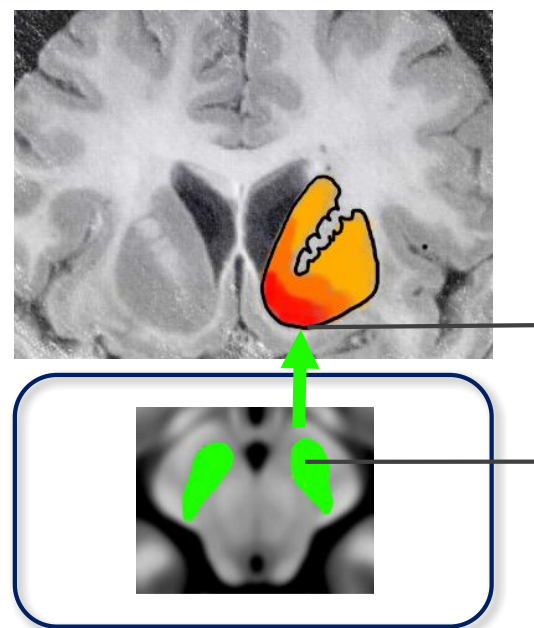

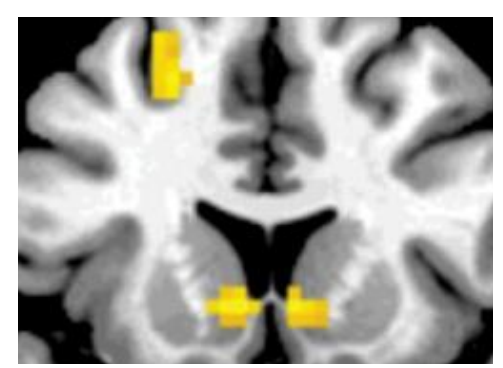

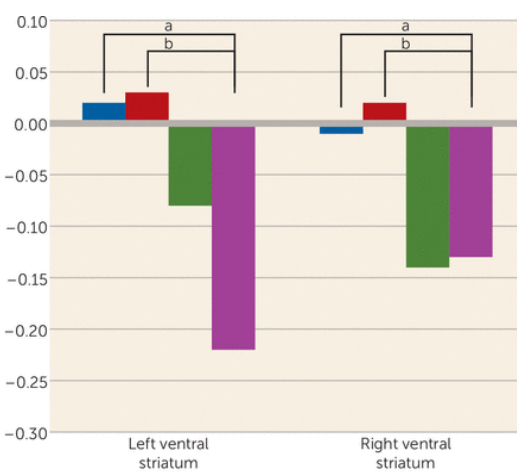

No depressive symptoms $(\mathrm{N}=535)$ Low mood ( $\mathrm{N}=509)$

Anhedonia $(\mathrm{N}=72)$

Low mood and anhedonia ( $N=182)$

\section{Figure 3 | Brain regions implicated in motivation, apathy and anhedonia}

A Frontostriatal circuits implicated in motivation to act for rewards and effort-based decision making include the dopaminergic projection from the ventral tegmental area (VTA) to the ventral striatum which includes the nucleus accumbens. Striatal regions project, via the thalamus, to different parts of the medial prefrontal cortex, including the ventromedial prefrontal cortex (vmPFC), dorsomedial prefrontal cortex (dmPFC) and the anterior cingulate cortex (ACC). These regions in turn project back to the basal ganglia. (Adapted from ${ }^{173}$ ). B Ventral striatal activation (BOLD response) in response to anticipation of reward on a monetary incentive task is reduced in adolescents with depressive symptoms, especially anhedonia. ${ }^{107}$ The graph shows normalized levels of activation in different groups of individuals. 


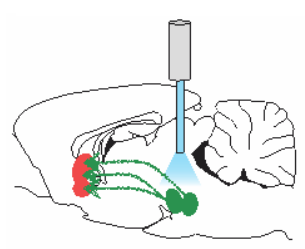

Stimulation of dopamine neurons

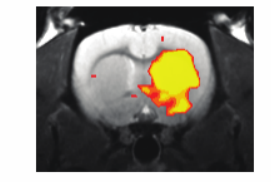

4 Reward-seeking

4 BOLD activity

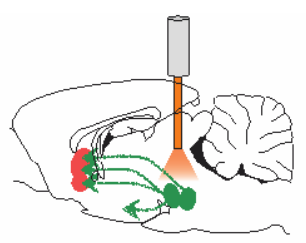

Silencing of dopamine neurons

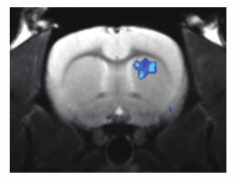

Reward-seeking

BOLD activity

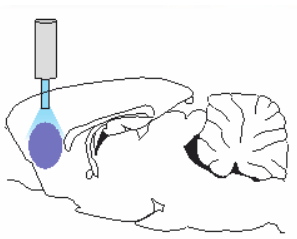

Elevated cortical excitability

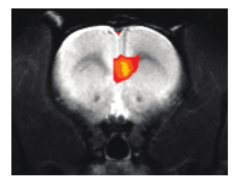

$\downarrow$ Reward-seeking

Asynchrony

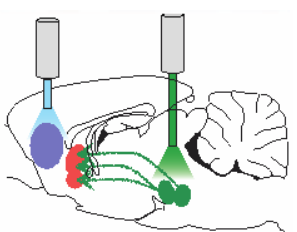

Top-down control of striatal dopamine signaling

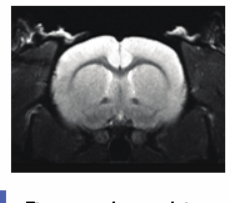

$\downarrow$ Reward-seeking

$\downarrow$ Dopamine response

Figure 4 | Effects of optogenetic stimulation of dopamine VTA neurons blunted by medial prefrontal stimulation

Stimulation of DA neurons in the VTA (green) led to increased BOLD activity in the striatum (orange) and animals choosing to work for such stimulation. This effect appeared to be dopaminergic because a DA receptor antagonist blocked both effects. Optogenetic silencing of VTA DA neurons led to reduced rewardseeking and striatal BOLD signal. Focally increased medial prefrontal cortex ( MPFC) stimulation that altered neural synchrony also suppressed reward seeking and blunted the effects of optogenetic VTA DA stimulation, both on striatal BOLD response and reward-seeking. ${ }^{94}$ 


\section{References}

1. Caeiro, L., Ferro, J. M. \& Costa, J. Apathy Secondary to Stroke: A Systematic Review and Meta-Analysis. Cerebrovasc. Dis. 35, 23-39 (2013).

2. Starkstein, S. E. \& Pahissa, J. Apathy Following Traumatic Brain Injury. Psychiatr. Clin. North Am. 37, 103-112 (2014).

3. Seel, R. T. et al. Depression after traumatic brain injury: a National Institute on Disability and Rehabilitation Research Model Systems multicenter investigation. Arch. Phys. Med. Rehabil. 84, 177-84 (2003).

4. Zhao, Q.-F. et al. The prevalence of neuropsychiatric symptoms in Alzheimer's disease: Systematic review and meta-analysis. J. Affect. Disord. 190, 264-271 (2016).

5. Lopez, O. L. et al. Psychiatric Symptoms Vary With the Severity of Dementia in Probable Alzheimer's Disease. J. Neuropsychiatry Clin. Neurosci. 15, 346-353 (2003).

6. den Brok, M. G. H. E. et al. Apathy in Parkinson's disease: A systematic review and meta-analysis. Mov. Disord. 30, 759-769 (2015).

7. Lemke, M. R., Brecht, H. M., Koester, J., Kraus, P. H. \& Reichmann, H. Anhedonia, Depression, and Motor Functioning in Parkinson's Disease During Treatment With Pramipexole. J. Neuropsychiatry Clin. Neurosci. 17, 214-220 (2005).

8. Staekenborg, S. S. et al. Behavioural and psychological symptoms in vascular dementia; differences between small- and large-vessel disease. J. Neurol. Neurosurg. Psychiatry 81, 547-551 (2010).

9. Chow, T. W. et al. Apathy symptom profile and behavioral associations in frontotemporal dementia vs dementia of Alzheimer type. Arch. Neurol. 66, 888-93 (2009).

10. van Duijn, E. et al. Neuropsychiatric symptoms in a European Huntington's disease cohort (REGISTRY). J. Neurol. Neurosurg. Psychiatry 85, 1411-1418 (2014).

11. Pelizza, L. \& Ferrari, A. Anhedonia in schizophrenia and major depression: state or trait? Ann. Gen. Psychiatry 8, 22 (2009).

12. Yuen, G. S. et al. Apathy in late-life depression: common, persistent, and disabling. Am. J. Geriatr. Psychiatry 23, 488-94 (2015).

13. Horan, W. P., Kring, A. M. \& Blanchard, J. J. Anhedonia in Schizophrenia: A Review of Assessment Strategies. Schizophr. Bull. 32, 259-273 (2006).

14. Yazbek, H. et al. The Lille Apathy Rating Scale (LARS): exploring its psychometric properties in schizophrenia. Schizophr. Res. 157, 278-84 (2014).

15. Brown, R. G. \& Pluck, G. Negative symptoms: the 'pathology' of motivation and goal-directed behaviour. Trends Neurosci. 23, 412-7 (2000).

16. Prange, S. et al. Historical crossroads in the conceptual delineation of apathy in Parkinson's disease. Brain (2018). doi:10.1093/brain/awx362

17. Starkstein, S. E. \& Leentjens, A. F. G. The nosological position of apathy in clinical practice. J. Neurol. Neurosurg. Psychiatry 79, 1088-1092 (2008).

18. Lanctôt, K. L. et al. Apathy associated with neurocognitive disorders: Recent progress and future directions. Alzheimer's Dement. 13, 84-100 (2017).

19. Robert, P. et al. Proposed diagnostic criteria for apathy in Alzheimer's disease and other neuropsychiatric disorders. Eur. Psychiatry 24, 98-104 (2009).

20. Thomsen, K. R., Whybrow, P. C. \& Kringelbach, M. L. Reconceptualizing anhedonia: novel perspectives on balancing the pleasure networks in the human brain. Front.

Behav. Neurosci. 9, 49 (2015). 
21. Foussias, G. \& Remington, G. Negative symptoms in schizophrenia: avolition and Occam's razor. Schizophr. Bull. 36, 359-69 (2010).

22. Treadway, M. T. \& Zald, D. H. Reconsidering anhedonia in depression: lessons from translational neuroscience. Neurosci. Biobehav. Rev. 35, 537-55 (2011).

23. Berridge, K. C. \& Robinson, T. E. Parsing reward. Trends Neurosci. 26, 507-513 (2003).

24. Barch, D. M., Pagliaccio, D. \& Luking, K. Mechanisms Underlying Motivational Deficits in Psychopathology: Similarities and Differences in Depression and Schizophrenia. Curr. Top. Behav. Neurosci. 27, 411-49 (2016).

25. Der-Avakian, A., Barnes, S. A., Markou, A. \& Pizzagalli, D. A. in Current topics in behavioral neurosciences 28, 231-262 (2015).

26. Salamone, J. D., Yohn, S. E., López-Cruz, L., San Miguel, N. \& Correa, M. Activational and effort-related aspects of motivation: neural mechanisms and implications for psychopathology. Brain 139, 1325-1347 (2016).

27. Le Heron, C., Apps, M. A. J. \& Husain, M. The anatomy of apathy: A neurocognitive framework for amotivated behaviour. Neuropsychologia (2017). doi:10.1016/j.neuropsychologia.2017.07.003

28. Marin, R. S. Apathy: a neuropsychiatric syndrome. J. Neuropsychiatry Clin. Neurosci. 3, 243-254 (1991).

29. Starkstein, S. E., Petracca, G., Chemerinski, E. \& Kremer, J. Syndromic Validity of Apathy in Alzheimer's Disease. Am. J. Psychiatry 158, 872-877 (2001).

30. Sockeel, P. et al. The Lille apathy rating scale (LARS), a new instrument for detecting and quantifying apathy: validation in Parkinson's disease. J. Neurol. Neurosurg. Psychiatry 77, 579-584 (2006).

31. Ang, Y.-S., Lockwood, P., Apps, M. A. J., Muhammed, K. \& Husain, M. Distinct Subtypes of Apathy Revealed by the Apathy Motivation Index. PLoS One 12, e0169938 (2017).

32. Németh, G., Hegedüs, K. \& Molnár, L. Akinetic mutism associated with bicingular lesions: clinicopathological and functional anatomical correlates. Eur. Arch. Psychiatry Neurol. Sci. 237, 218-22 (1988).

33. Rizvi, S. J. et al. Development and validation of the Dimensional Anhedonia Rating Scale (DARS) in a community sample and individuals with major depression. Psychiatry Res. 229, 109-119 (2015).

34. Shankman, S. A. et al. in Anhedonia: A Comprehensive Handbook Volume I 3-22 (Springer Netherlands, 2014). doi:10.1007/978-94-017-8591-4_1

35. Assogna, F., Cravello, L., Caltagirone, C. \& Spalletta, G. Anhedonia in Parkinson's disease: A systematic review of the literature. Mov. Disord. 26, 1825-1834 (2011).

36. Blanchard, J. J. \& Cohen, A. S. The Structure of Negative Symptoms Within Schizophrenia: Implications for Assessment. Schizophr. Bull. 32, 238-245 (2006).

37. Foussias, G., Agid, O., Fervaha, G. \& Remington, G. Negative symptoms of schizophrenia: clinical features, relevance to real world functioning and specificity versus other CNS disorders. Eur. Neuropsychopharmacol. 24, 693-709 (2014).

38. Kaiser, S. et al. Individual negative symptoms and domains - Relevance for assessment, pathomechanisms and treatment. Schizophr. Res. 186, 39-45 (2017).

39. Bischof, M. et al. The brief negative symptom scale: validation of the German translation and convergent validity with self-rated anhedonia and observer-rated apathy. BMC Psychiatry 16, 415 (2016).

40. Hartmann, M. N. et al. Apathy in schizophrenia as a deficit in the generation of 
options for action. J. Abnorm. Psychol. 124, 309-318 (2015).

41. Isella, V. et al. Physical anhedonia in Parkinson's disease. J. Neurol. Neurosurg. Psychiatry 74, 1308-11 (2003).

42. Skorvanek, M. et al. The associations between fatigue, apathy, and depression in Parkinson's disease. Acta Neurol. Scand. 131, 80-87 (2015).

43. Lampe, I. K., Kahn, R. S. \& Heeren, T. J. Apathy, anhedonia, and psychomotor retardation in elderly psychiatric patients and healthy elderly individuals. J. Geriatr. Psychiatry Neurol. 14, 11-6 (2001).

44. Brodaty, H., Altendorf, A., Withall, A. \& Sachdev, P. Do people become more apathetic as they grow older? A longitudinal study in healthy individuals. Int. psychogeriatrics 22, 426-36 (2010).

45. Bonnelle, V., Manohar, S., Behrens, T. \& Husain, M. Individual Differences in Premotor Brain Systems Underlie Behavioral Apathy. Cereb. Cortex 26, bhv247 (2016).

46. Lavretsky, H. et al. The MRI brain correlates of depressed mood, anhedonia, apathy, and anergia in older adults with and without cognitive impairment or dementia. Int. J. Geriatr. Psychiatry 23, 1040-1050 (2008).

47. Grool, A. M. et al. Structural MRI correlates of apathy symptoms in older persons without dementia: AGES-Reykjavik Study. Neurology 82, 1628-1635 (2014).

48. Kawagoe, T., Onoda, K. \& Yamaguchi, S. Apathy and Executive Function in Healthy Elderly-Resting State fMRI Study. Front. Aging Neurosci. 9, 124 (2017).

49. Rzepa, E., Fisk, J. \& McCabe, C. Blunted neural response to anticipation, effort and consummation of reward and aversion in adolescents with depression symptomatology. J. Psychopharmacol. 31, 303-311 (2017).

50. Pessiglione, M., Vinckier, F., Bouret, S., Daunizeau, J. \& Le Bouc, R. Why not try harder? Computational approach to motivation deficits in neuro-psychiatric diseases. Brain (2017). doi:10.1093/brain/awX278

51. Sinha, N., Manohar, S. \& Husain, M. Impulsivity and apathy in Parkinson's disease. J. Neuropsychol. 7, 255-283 (2013).

52. Ang, Y.-S. et al. Dopamine modulates choices for self-generated behaviour. Curr. Biol. In Press, (2018).

53. Robinson, G., Shallice, T., Bozzali, M. \& Cipolotti, L. The differing roles of the frontal cortex in fluency tests. Brain 135, 2202-2214 (2012).

54. Neuroeconomics. Glimcher, Ernst Fehr : decision making and the brain. (Elsevier Academic Press, 2014).

55. Le Bouc, R. et al. Computational Dissection of Dopamine Motor and Motivational Functions in Humans. J. Neurosci. 36, 6623-6633 (2016).

56. Le Heron, C. et al. Distinct effects of apathy and dopamine on effort-based decision making in Parkinson's disease. Brain

57. Brehm, J. W. \& Self, E. A. The Intensity of Motivation. Annu. Rev. Psychol. 40, 109131 (1989).

58. Muhammed, K. et al. Reward sensitivity deficits modulated by dopamine are associated with apathy in Parkinson's disease. Brain 139, 2706-2721 (2016).

59. Salamone, J. D., Cousins, M. S. \& Bucher, S. Anhedonia or anergia? Effects of haloperidol and nucleus accumbens dopamine depletion on instrumental response selection in a T-maze cost/benefit procedure. Behav. Brain Res. 65, 221-9 (1994).

6o. Treadway, M. T., Bossaller, N. A., Shelton, R. C. \& Zald, D. H. Effort-based decision- 
making in major depressive disorder: A translational model of motivational anhedonia. J. Abnorm. Psychol. 121, 553-558 (2012).

61. Chong, T. T.-J. et al. Dopamine enhances willingness to exert effort for reward in Parkinson's disease. Cortex 69, 40-46 (2015).

62. Murray, G. K. et al. Incentive motivation in first-episode psychosis: A behavioural study. BMC Psychiatry 8, 34 (2008).

63. Hosking, J. G., Cocker, P. J. \& Winstanley, C. A. Dissociable Contributions of Anterior Cingulate Cortex and Basolateral Amygdala on a Rodent Cost/Benefit DecisionMaking Task of Cognitive Effort. Neuropsychopharmacology 39, 1558-1567 (2014).

64. Schmidt, L., Lebreton, M., Cléry-Melin, M.-L., Daunizeau, J. \& Pessiglione, M. Neural Mechanisms Underlying Motivation of Mental Versus Physical Effort. PLoS Biol. 10, e1001266 (2012).

65. Chong, T. T.-J. et al. Neurocomputational mechanisms underlying subjective valuation of effort costs. PLOS Biol. 15, e1002598 (2017).

66. Berridge, K. C. \& Robinson, T. E. What is the role of dopamine in reward: hedonic impact, reward learning, or incentive salience? Brain Res. Brain Res. Rev. 28, 309-69 (1998).

67. Huys, Q. J. M. et al. The specificity of Pavlovian regulation is associated with recovery from depression. Psychol. Med. 46, 1027-1035 (2016).

68. Willner, P. The chronic mild stress (CMS) model of depression: History, evaluation and usage. Neurobiol. Stress 6, 78-93 (2017).

69. Huys, Q. J. M., Daw, N. D. \& Dayan, P. Depression: a decision-theoretic analysis. Annu. Rev. Neurosci. 38, 1-23 (2015).

70. Bechara, A., Damasio, H., Tranel, D. \& Damasio, A. R. Deciding advantageously before knowing the advantageous strategy. Science 275, 1293-5 (1997).

71. Evens, R., Hoefler, M., Biber, K. \& Lueken, U. The lowa Gambling Task in Parkinson's disease: A meta-analysis on effects of disease and medication. Neuropsychologia 91, 163-172 (2016).

72. Adams, R. A., Huys, Q. J. M. \& Roiser, J. P. Computational Psychiatry: towards a mathematically informed understanding of mental illness. J. Neurol. Neurosurg. Psychiatry 87, jnnp-2015-310737 (2015).

73. Henriques, J. B., Glowacki, J. M. \& Davidson, R. J. Reward fails to alter response bias in depression. J. Abnorm. Psychol. 103, 460-6 (1994).

74. Huys, Q. J., Pizzagalli, D. A., Bogdan, R. \& Dayan, P. Mapping anhedonia onto reinforcement learning: a behavioural meta-analysis. Biol. Mood Anxiety Disord. 3, 12 (2013).

75. Collins, A. G. E., Brown, J. K., Gold, J. M., Waltz, J. A. \& Frank, M. J. Working Memory Contributions to Reinforcement Learning Impairments in Schizophrenia. J. Neurosci. 34, 13747-13756 (2014).

76. Meyniel, F. et al. A specific role for serotonin in overcoming effort cost. Elife 5, (2016).

77. Levy, R. \& Dubois, B. Apathy and the Functional Anatomy of the Prefrontal CortexBasal Ganglia Circuits. Cereb. Cortex 16, 916-928 (2005).

78. Barris, R. W. \& Schuman, H. R. Bilateral anterior cingulate gyrus lesions; syndrome of the anterior cingulate gyri. Neurology 3, 44-52 (1953).

79. Laplane, D. et al. Obsessive-compulsive and other behavioural changes with bilateral basal ganglia lesions. A neuropsychological, magnetic resonance imaging and 
positron tomography study. Brain 112 ( Pt 3), 699-725 (1989).

8o. Adam, R. et al. Dopamine reverses reward insensitivity in apathy following globus pallidus lesions. Cortex 49, 1292-1303 (2013).

81. Kang, S. Y. \& Kim, J. S. Anterior cerebral artery infarction: Stroke mechanism and clinical-imaging study in 100 patients. Neurology 70, 2386-2393 (2008).

82. Manohar, S. G. \& Husain, M. Human ventromedial prefrontal lesions alter incentivisation by reward. Cortex. 76, 104-20 (2016).

83. Heath, R. G. Pleasure and brain activity in man. Deep and surface electroencephalograms during orgasm. J. Nerv. Ment. Dis. 154, 3-18 (1972).

84. Berridge, K. C. \& Kringelbach, M. L. Pleasure Systems in the Brain. Neuron 86, 646664 (2015).

85. Parvizi, J., Rangarajan, V., Shirer, W. R., Desai, N. \& Greicius, M. D. The will to persevere induced by electrical stimulation of the human cingulate gyrus. Neuron 80 , 1359-67 (2013).

86. Mayberg, H. S. et al. Deep Brain Stimulation for Treatment-Resistant Depression. Neuron 45, 651-66o (2005).

87. Holtzheimer, P. E. et al. Subcallosal cingulate deep brain stimulation for treatmentresistant depression: a multisite, randomised, sham-controlled trial. The Lancet Psychiatry (2017). doi:10.1016/S2215-0366(17)30371-1

88. Rudebeck, P. H., Walton, M. E., Smyth, A. N., Bannerman, D. M. \& Rushworth, M. F. S. Separate neural pathways process different decision costs. Nat. Neurosci. 9, 11618 (2006).

89. Walton, M. E., Kennerley, S. W., Bannerman, D. M., Phillips, P. E. M. \& Rushworth, M. F. S. Weighing up the benefits of work: behavioral and neural analyses of effortrelated decision making. Neural Netw. 19, 1302-14 (2006).

90. Floresco, S. B. \& Ghods-Sharifi, S. Amygdala-prefrontal cortical circuitry regulates effort-based decision making. Cereb. Cortex 17, 251-60 (2007).

91. Hauber, W. \& Sommer, S. Prefrontostriatal Circuitry Regulates Effort-Related Decision Making. Cereb. Cortex 19, 2240-2247 (2009).

92. Worbe, Y. et al. Behavioral and Movement Disorders Induced by Local Inhibitory Dysfunction in Primate Striatum. Cereb. Cortex 19, 1844-1856 (2009).

93. Wise, R. A. Addictive Drugs and Brain Stimulation Reward. Annu. Rev. Neurosci. 19, 319-340 (1996).

94. Ferenczi, E. A. et al. Prefrontal cortical regulation of brainwide circuit dynamics and reward-related behavior. Science (80-. ). 351, aac9698-aac9698 (2016).

95. Croxson, P. L., Walton, M. E., O’Reilly, J. X., Behrens, T. E. J. \& Rushworth, M. F. S. Effort-Based Cost-Benefit Valuation and the Human Brain. J. Neurosci. 29, 45314541 (2009).

96. Skvortsova, V., Palminteri, S. \& Pessiglione, M. Learning to minimize efforts versus maximizing rewards: computational principles and neural correlates. J. Neurosci. 34, 15621-30 (2014).

97. Klein-Flugge, M. C., Kennerley, S. W., Friston, K. \& Bestmann, S. Neural Signatures of Value Comparison in Human Cingulate Cortex during Decisions Requiring an Effort-Reward Trade-off. J. Neurosci. 36, 10002-10015 (2016).

98. Zenon, A., Sidibe, M. \& Olivier, E. Disrupting the Supplementary Motor Area Makes Physical Effort Appear Less Effortful. J. Neurosci. 35, 8737-8744 (2015).

99. Kroemer, N. B. et al. Balancing reward and work: anticipatory brain activation in 
NAcc and VTA predict effort differentially. Neuroimage 102 Pt 2, 510-9 (2014).

100. Kurniawan, I. T., Guitart-Masip, M., Dayan, P. \& Dolan, R. J. Effort and Valuation in the Brain: The Effects of Anticipation and Execution. J. Neurosci. 33, 6160-6169 (2013).

101. Lau, H. C., Rogers, R. D., Haggard, P. \& Passingham, R. E. Attention to Intention. Science (80-. ). 303, 1208-1210 (2004).

102. Nachev, P., Kennard, C. \& Husain, M. Functional role of the supplementary and presupplementary motor areas. Nat. Rev. Neurosci. 9, 856-869 (2008).

103. Pizzagalli, D. A. et al. Reduced Caudate and Nucleus Accumbens Response to Rewards in Unmedicated Individuals With Major Depressive Disorder. Am. J. Psychiatry 166, 702-710 (2009).

104. Knutson, B., Bhanji, J. P., Cooney, R. E., Atlas, L. Y. \& Gotlib, I. H. Neural Responses to Monetary Incentives in Major Depression. Biol. Psychiatry 63, 686-692 (2008).

105. Gorka, S. M. et al. Neural response to reward anticipation in those with depression with and without panic disorder. J. Affect. Disord. 164, 50-56 (2014).

106. Rutledge, R. B. et al. Association of Neural and Emotional Impacts of Reward Prediction Errors With Major Depression. JAMA Psychiatry 74, 790 (2017).

107. Stringaris, A. et al. The Brain's Response to Reward Anticipation and Depression in Adolescence: Dimensionality, Specificity, and Longitudinal Predictions in a Community-Based Sample. Am. J. Psychiatry 172, 1215-1223 (2015).

108. Ziauddeen, H. \& Murray, G. K. The relevance of reward pathways for schizophrenia. Curr. Opin. Psychiatry 23, 91-96 (2010).

109. Zhang, B. et al. Mapping anhedonia-specific dysfunction in a transdiagnostic approach: an ALE meta-analysis. Brain Imaging Behav. 10, 920-939 (2016).

110. Howe, M. W., Tierney, P. L., Sandberg, S. G., Phillips, P. E. M. \& Graybiel, A. M. Prolonged dopamine signalling in striatum signals proximity and value of distant rewards. Nature 500, 575-579 (2013).

111. Robbins, T. W., Roberts, D. C. \& Koob, G. F. Effects of d-amphetamine and apomorphine upon operant behavior and schedule-induced licking in rats with 6hydroxydopamine-induced lesions of the nucleus accumbens. J. Pharmacol. Exp. Ther. 224, 662-73 (1983).

112. Taylor, J. R. \& Robbins, T. W. Enhanced behavioural control by conditioned reinforcers following microinjections of d-amphetamine into the nucleus accumbens. Psychopharmacology (Berl). 84, 405-12 (1984).

113. Cador, M., Taylor, J. R. \& Robbins, T. W. Potentiation of the effects of reward-related stimuli by dopaminergic-dependent mechanisms in the nucleus accumbens. Psychopharmacology (Berl). 104, 377-85 (1991).

114. Salamone, J. D. \& Correa, M. The mysterious motivational functions of mesolimbic dopamine. Neuron 76, 470-85 (2012).

115. Schultz, W., Dayan, P. \& Montague, P. R. A neural substrate of prediction and reward. Science 275, 1593-9 (1997).

116. Chang, C. Y. et al. Brief optogenetic inhibition of dopamine neurons mimics endogenous negative reward prediction errors. Nat. Neurosci. 19, 111-6 (2016).

117. Steinberg, E. E. et al. A causal link between prediction errors, dopamine neurons and learning. Nat. Neurosci. 16, 966-973 (2013).

118. Niv, Y., Daw, N. D., Joel, D. \& Dayan, P. Tonic dopamine: opportunity costs and the control of response vigor. Psychopharmacology (Berl). 191, 507-520 (2007). 
119. Hamid, A. A. et al. Mesolimbic dopamine signals the value of work. Nat. Neurosci. 19, 117-126 (2015).

120. Syed, E. C. J. et al. Action initiation shapes mesolimbic dopamine encoding of future rewards. Nat. Neurosci. 19, 34-36 (2015).

121. Collins, A. G. E. \& Frank, M. J. Surprise! Dopamine signals mix action, value and error. Nat. Neurosci. 19, 3-5 (2016).

122. Martins, D., Mehta, M. A. \& Prata, D. The 'highs and lows' of the human brain on dopaminergics: Evidence from neuropharmacology. Neurosci. Biobehav. Rev. 80, 351-371 (2017).

123. Leyton, M. et al. Decreasing Amphetamine-Induced Dopamine Release by Acute Phenylalanine/Tyrosine Depletion: A PET/[11C]Raclopride Study in Healthy Men. Neuropsychopharmacology 29, 427-32 (2003).

124. Roiser, J. P. et al. The Subjective and Cognitive Effects of Acute Phenylalanine and Tyrosine Depletion in Patients Recovered from Depression. Neuropsychopharmacology 30, 775-85 (2005).

125. Cox, S. M. L. et al. Striatal $D_{1}$ and $D_{2}$ signaling differentially predict learning from positive and negative outcomes. Neuroimage 109, 95-101 (2015).

126. Bjork, J. M., Grant, S. J., Chen, G. \& Hommer, D. W. Dietary Tyrosine/Phenylalanine Depletion Effects on Behavioral and Brain Signatures of Human Motivational Processing. Neuropsychopharmacology 39, 595-604 (2014).

127. Guitart-Masip, M. et al. Action controls dopaminergic enhancement of reward representations. Proc. Natl. Acad. Sci. 109, 7511-7516 (2012).

128. Zenon, A., Devesse, S. \& Olivier, E. Dopamine Manipulation Affects Response Vigor Independently of Opportunity Cost. J. Neurosci. 36, 9516-9525 (2016).

129. Rutledge, R. B., Skandali, N., Dayan, P. \& Dolan, R. J. Dopaminergic Modulation of Decision Making and Subjective Well-Being. J. Neurosci. 35, 9811-9822 (2015).

130. Chowdhury, R. et al. Dopamine restores reward prediction errors in old age. Nat. Neurosci. 16, 648-653 (2013).

131. Argyropoulos, S. V \& Nutt, D. J. Anhedonia revisited: Is there a role for dopaminetargeting drugs for depression? J. Psychopharmacol. 27, 869-877 (2013).

132. Di Giannantonio, M. \& Martinotti, G. Anhedonia and major depression: The role of agomelatine. Eur. Neuropsychopharmacol. 22, $\mathrm{S}_{505}-\mathrm{S}_{510}$ (2012).

133. Thobois, S. et al. Parkinsonian apathy responds to dopaminergic stimulation of D2/D3 receptors with piribedil. Brain 136, 1568-1577 (2013).

134. Devos, D. et al. Rivastigmine in apathetic but dementia and depression-free patients with Parkinson's disease: a double-blind, placebo-controlled, randomised clinical trial. J. Neurol. Neurosurg. Psychiatry 85, 668-674 (2014).

135. McGirr, A. et al. A systematic review and meta-analysis of randomized, double-blind, placebo-controlled trials of ketamine in the rapid treatment of major depressive episodes. Psychol. Med. 45, 693-704 (2015).

136. Kokkinou, M., Ashok, A. H. \& Howes, O. D. The effects of ketamine on dopaminergic function: meta-analysis and review of the implications for neuropsychiatric disorders. Mol. Psychiatry (2017). doi:10.1038/mp.2017.190

137. Lally, N. et al. Anti-anhedonic effect of ketamine and its neural correlates in treatment-resistant bipolar depression. Transl. Psychiatry 4, e469 (2014).

138. Peciña, M. et al. Striatal dopamine $D_{2} / 3$ receptor-mediated neurotransmission in major depression: Implications for anhedonia, anxiety and treatment response. Eur. 
Neuropsychopharmacol. 27, 977-986 (2017).

139. Remy, P., Doder, M., Lees, A., Turjanski, N. \& Brooks, D. Depression in Parkinson's disease: loss of dopamine and noradrenaline innervation in the limbic system. Brain 128, 1314-1322 (2005).

140. Sarchiapone, M. et al. Dopamine transporter binding in depressed patients with anhedonia. Psychiatry Res. Neuroimaging 147, 243-248 (2006).

141. Bloomfield, M. A. P., Morgan, C. J. A., Kapur, S., Curran, H. V. \& Howes, O. D. The link between dopamine function and apathy in cannabis users: an [18F]-DOPA PET imaging study. Psychopharmacology (Berl). 231, 2251-2259 (2014).

142. Felger, J. C. \& Treadway, M. T. Inflammation Effects on Motivation and Motor Activity: Role of Dopamine. Neuropsychopharmacology 42, 216-241 (2017).

143. Felger, J. C. \& Miller, A. H. Cytokine effects on the basal ganglia and dopamine function: The subcortical source of inflammatory malaise. Front. Neuroendocrinol. 33, 315-327 (2012).

144. Kapur, S. \& Remington, G. Serotonin-dopamine interaction and its relevance to schizophrenia. Am. J. Psychiatry 153, 466-476 (1996).

145. Bailey, M. R. et al. The effects of pharmacological modulation of the serotonin $2 C$ receptor on goal-directed behavior in mice. Psychopharmacology (Berl). 233, 615-624 (2016).

146. Deakin, J. F. W. \& Graeff, F. G. 5-HT and mechanisms of defence. J.

Psychopharmacol. 5, 305-315 (1991).

147. Maier, S. F. \& Watkins, L. R. Stressor controllability and learned helplessness: The roles of the dorsal raphe nucleus, serotonin, and corticotropin-releasing factor. Neurosci. Biobehav. Rev. 29, 829-841 (2005).

148. Daw, N. D., Kakade, S. \& Dayan, P. Opponent interactions between serotonin and dopamine. Neural Netw. 15, 603-16

149. Boureau, Y.-L. \& Dayan, P. Opponency Revisited: Competition and Cooperation Between Dopamine and Serotonin. Neuropsychopharmacology 36, 74-97 (2011).

150. Kranz, G. S., Kasper, S. \& Lanzenberger, R. Reward and the serotonergic system. Neuroscience 166, 1023-1035 (2010).

151. Nakamura, K., Matsumoto, M. \& Hikosaka, O. Reward-Dependent Modulation of Neuronal Activity in the Primate Dorsal Raphe Nucleus. J. Neurosci. 28, 5331-5343 (2008).

152. Fonseca, M. S., Murakami, M. \& Mainen, Z. F. Activation of Dorsal Raphe Serotonergic Neurons Promotes Waiting but Is Not Reinforcing. Curr. Biol. 25, 306315 (2015).

153. Luo, M., Zhou, J. \& Liu, Z. Reward processing by the dorsal raphe nucleus: $5-\mathrm{HT}$ and beyond. Learn. Mem. 22, 452-60 (2015).

154. Faulkner, P. \& Deakin, J. F. W. The role of serotonin in reward, punishment and behavioural inhibition in humans: Insights from studies with acute tryptophan depletion. Neurosci. Biobehav. Rev. 46, 365-378 (2014).

155. Macoveanu, J. Serotonergic modulation of reward and punishment: Evidence from pharmacological fMRI studies. Brain Res. 1556, 19-27 (2014).

156. Seymour, B., Daw, N. D., Roiser, J. P., Dayan, P. \& Dolan, R. Serotonin Selectively Modulates Reward Value in Human Decision-Making. J. Neurosci. 32, 5833-5842 (2012).

157. Cools, R. et al. Tryptophan Depletion Disrupts the Motivational Guidance of Goal- 
Directed Behavior as a Function of Trait Impulsivity. Neuropsychopharmacology 30, 1362-73 (2005).

158. Maillet, A. et al. The prominent role of serotonergic degeneration in apathy, anxiety and depression in de novo Parkinson's disease. Brain 139, 2486-2502 (2016).

159. Da Silva, S. et al. Investigating consummatory and anticipatory pleasure across motivation deficits in schizophrenia and healthy controls. Psychiatry Res. 254, 112117 (2017).

16o. Richards, D. A. et al. Cost and Outcome of Behavioural Activation versus Cognitive Behavioural Therapy for Depression (COBRA): a randomised, controlled, noninferiority trial. Lancet (London, England) 388, 871-80 (2016).

161. Lutgens, D., Gariepy, G. \& Malla, A. Psychological and psychosocial interventions for negative symptoms in psychosis: systematic review and meta-analysis. Br. J. Psychiatry 210, 324-332 (2017).

162. Rizvi, S. J., Pizzagalli, D. A., Sproule, B. A. \& Kennedy, S. H. Assessing anhedonia in depression: Potentials and pitfalls. Neurosci. Biobehav. Rev. 65, 21-35 (2016).

163. Strauss, G. P. \& Gold, J. M. A Psychometric Comparison of the Clinical Assessment Interview for Negative Symptoms and the Brief Negative Symptom Scale. Schizophr. Bull. 42, 1384-1394 (2016).

164. Kaji, Y. \& Hirata, K. Apathy and anhedonia in Parkinson's disease. ISRN Neurol. 2011, 219427 (2011).

165. Haarasilta, L., Marttunen, M., Kaprio, J. \& Aro, H. The 12-month prevalence and characteristics of major depressive episode in a representative nationwide sample of adolescents and young adults. Psychol. Med. 31, 1169-79 (2001).

166. Kiwanuka, J. N., Strauss, G. P., McMahon, R. P. \& Gold, J. M. Psychological predictors of functional outcome in people with schizophrenia. Schizophr. Res. 157, 299-304 (2014).

167. Adams, R. A., Huys, Q. J. M. \& Roiser, J. P. Computational Psychiatry: Towards a mathematically informed understanding of mental illness. J. Neurol. Neurosurg. Psychiatry 87, (2016).

168. Daw, N. D. in Decision Making, Affect, and Learning 3-38 (Oxford University Press, 2011). doi:10.1093/acprof:0so/9780199600434.003.0001

169. Anticevic, A. \& Murray, J. D. (John D. Computational psychiatry : mathematical modeling of mental illness.

170. Gelman, A. \& Shalizi, C. R. Philosophy and the practice of Bayesian statistics. Br. J. Math. Stat. Psychol. 66, 8-38 (2013).

171. Pessiglione, M., Seymour, B., Flandin, G., Dolan, R. J. \& Frith, C. D. Dopaminedependent prediction errors underpin reward-seeking behaviour in humans. Nature 442, 1042-1045 (2006).

172. Sutton, R. S. \& Barto, A. G. Reinforcement learning : an introduction. (MIT Press, 1998).

173. Haber, S. N. \& Knutson, B. The reward circuit: linking primate anatomy and human imaging. Neuropsychopharmacology 35, 4-26 (2010).

\section{Starred references}

Treadway MT, Zald DH. Reconsidering anhedonia in depression: lessons from translational 
neuroscience. Neurosci Biobehav Rev 2011; 35: 537-55.

A helpful overview providing mechanistic insights on anhedonia in human depression.

Barch DM, Pagliaccio D, Luking K. Mechanisms Underlying Motivational Deficits in

Psychopathology: Similarities and Differences in Depression and Schizophrenia. Curr Top

Behav Neurosci 2016; 27: 411-49.

Comprehensive current review of evidence for possible underlying behavioral components of amotivation across depression and schizophrenia.

Der-Avakian A, Barnes SA, Markou A, Pizzagalli DA. Translational Assessment of Reward and Motivational Deficits in Psychiatric Disorders. In: Current topics in behavioral neurosciences. 2015: 231-62.

Overview relating animal models to human disorders of motivation in psychiatric diagnoses.

Salamone JD, Yohn SE, López-Cruz L, San Miguel N, Correa M. Activational and effort-related aspects of motivation: neural mechanisms and implications for psychopathology. Brain 2016; 139: 1325-47.

Comprehensive review of animal and human experiments investigating the brain mechanisms of effort-related decision making for reward, and how these relate to anhedonia and apathy.

Le Heron C, Apps MAJ, Husain M. The anatomy of apathy: A neurocognitive framework for amotivated behaviour. Neuropsychologia 2017; published online July 8.

DOI:10.1016/j.neuropsychologia.2017.07.003.

A review of neuroimaging studies of apathy across neurological diseases demonstrating many common patterns of regional brain changes.

Hartmann MN, Kluge A, Kalis A, Mojzisch A, Tobler PN, Kaiser S. Apathy in schizophrenia as a deficit in the generation of options for action. J Abnorm Psychol 2015; 124: 309-18.

One of the few studies that have examined the possibility that a deficit in generating options for behaviour might be associated with apathy in humans.

Rizvi SJ, Pizzagalli DA, Sproule BA, Kennedy SH. Assessing anhedonia in depression: Potentials and pitfalls. Neurosci Biobehav Rev 2016; 65: 21-35.

Thorough review of questionnaire, interview and behavioral measurements commonly used to assess anhedonia in humans, and behavioral tests assessing related constructs in animals.

Le Bouc R, Rigoux L, Schmidt L, et al. Computational Dissection of Dopamine Motor and Motivational Functions in Humans. J Neurosci 2016; 36: 6623-33.

A study that combines empirical findings with computational modelling to pinpoint impaired reward processing in Parkinson's disease.

Berridge KC, Robinson TE. What is the role of dopamine in reward: hedonic impact, reward learning, or incentive salience? Brain Res Brain Res Rev 1998; 28: 309-69.

Landmark experimental paper examining the role of dopamine in appetitive and consummatory reward processing, providing clear evidence that the former, but not the latter, was disrupted by profound dopamine depletion.

Huys QJM, Daw ND, Dayan P. Depression: a decision-theoretic analysis. Annu Rev Neurosci 2015; 38: 1-23.

Clear explanation from a computational neuroscience perspective of the different aspects of motivational processing that may underlie depressive symptoms. 
Haber SN, Knutson B. The reward circuit: linking primate anatomy and human imaging.

Neuropsychopharmacology 2010; 35: 4-26.

Very helpful review that brings together animak and human studies on brain systems involved in reward processing.

Ferenczi EA, Zalocusky KA, Liston C, et al. Prefrontal cortical regulation of brainwide circuit dynamics and reward-related behavior. Science (80-) 2016; 351: aac9698-aac9698.

Pioneering experimental paper combining functional magnetic resonance imaging with simultaneous optogenetic stimulation of both dopamine and medial prefrontal cortex neurons. Stimulating dopamine neurons increased (and silencing reduced) ventral striatal responses and reward-seeking behavior, which was blunted by medial prefrontal stimulation.

Hamid AA, Pettibone JR, Mabrouk OS, et al. Mesolimbic dopamine signals the value of work. Nat Neurosci 2015; 19: 117-26.

In this investigation microdialysis and voltammetry were used to measure dopamine release in the nucleus accumbens. Dopamine levels covaried with reward rate and motivational vigor, and also encoded value. The authors conclude that dopamine conveys a signal regarding the available reward for investment of effort.

Syed ECJ, Grima LL, Magill PJ, Bogacz R, Brown P, Walton ME. Action initiation shapes mesolimbic dopamine encoding of future rewards. Nat Neurosci 2015; 19: 34-6.

This study used used fast scan cyclic voltametry to demonstrate that dopamine is released in the ventral striatum when animals are required to make an action to obtain a reward, and that dopamine release is attenuated when inhibition of movement is required.

Martins D, Mehta MA, Prata D. The 'highs and lows' of the human brain on dopaminergics: Evidence from neuropharmacology. Neurosci Biobehav Rev 2017; 80: 351-71.

Systematic review of behavioral and neural effects of dopamine manipulations in humans.

Felger JC, Treadway MT. Inflammation Effects on Motivation and Motor Activity: Role of Dopamine. Neuropsychopharmacology 2017; 42: 216-41.

Clear account of the links between the immune and dopamine systems, including how inflammation may result in a decrease in dopamine transmission, leading to symptoms related to amotivation. 